\title{
Characterization and Development of EST-SSR Markers from Transcriptome Sequences of Chrysanthemum (Chrysanthemum $\times$ morifolium Ramat.)
}

\author{
Min Fan, Yike Gao', Yaohui Gao, Zhiping Wu, Hua Liu, \\ and Qixiang Zhang \\ National Flower Engineering Research Centre, Beijing Key Laboratory of \\ Ornamental Plants Germplasm Innovation and Molecular Breeding, College \\ of Landscape Architecture of Beijing Forestry University, Beijing 100083, \\ China
}

Additional index words. Chrysanthemum, EST-SSR, Transferability, Functional annotation

\begin{abstract}
Simple sequence repeat (SSR) markers are valuable for genetic and breeding applications, but SSR resources for the ornamental genus chrysanthemum (Chrysanthemum $\times$ morifolium Ramat.) are still limited. Expressed sequence tags (ESTs) are sources of SSRs that represent an opportunity to develop SSRs to accelerate molecular breeding in chrysanthemum. In total, 4661 SSR loci were identified from 3823 SSR-containing unigenes in the chrysanthemum transcriptome with an average of one SSR per $6.98 \mathrm{~kb}$. Of these SSR sequences, trinucleotide repeats $(30.0 \%)$ predominated, followed by dinucleotide repeats $(17.9 \%)$. In total, 1584 primer pairs were subsequently synthesized. By screening the parents and six individuals of the $F_{1}$ progeny, $831(52.5 \%)$ valid EST-SSR markers were identified, of which $361(43.4 \%)$ were polymorphic. The annotation of unigenes containing polymorphic SSRs indicated that $330(93.5 \%)$ demonstrated significant homology to other plant protein sequences. Twenty-five polymorphic EST-SSR markers were further selected for transferability analysis and exhibited $93 \%$ amplification in six Ajania species and six other Chrysanthemum species. Based on genotyping of the 59 samples, neighbor-joining analysis revealed six phylogenetic groupings, which was confirmed by population structure analysis and principal component analysis (PCA). Phylogenetic relationships among the 59 samples revealed by SSRs were highly consistent with the traditional taxonomic classification of Chrysanthemum and Ajania. The polymorphism information content (PIC) values ranged from 0.29 to 0.86 , with a mean of 0.67 , indicating high levels of informativeness. This research reveals the SSR distribution characteristics of chrysanthemum and provides a large number of new EST-SSR markers for further genetic diversity studies, genetic mapping, and molecular marker-assisted selection breeding for chrysanthemum.
\end{abstract}

Chrysanthemums (Chrysanthemum $\times$ morifolium Ramat.) are common flowers that possess substantial aesthetic value. They are cultivated all over the world and are important economic ornamentals that comprise a considerable proportion of the flower industry in many southeast Asian and European countries (Zhang et al., 2011). Cultivated chrysanthemums are generally believed to be a hybrid complex derived from natural hybridization involving several species, such as Chrysanthemum vestitum, Chrysanthemum indicum, Chrysanthemum lavandulifolium, and Chrysanthemum

Received for publication 23 Oct. 2018. Accepted for publication 8 Jan. 2019

This study was funded by the National Natural Science Foundation of China (NSFC, 31770736) and the National High Technology Research and Development Program of China (863 Program, 2013AA102706).

${ }^{1}$ Corresponding author. E-mail: gaoyk@bjfu. edu.cn. zawadskii (Dai et al., 1998; Yang et al., 2006). They are classified as segmental allohexaploid with a most frequent somatic chromosome number of $54(2 n=6 x=54)$ (Klie et al., 2014) and an extremely large genome size of $\approx 9.4 \mathrm{~Gb}$ (http://data.kew.org/cvalues/). Cultivated chrysanthemums possess high genetic variation, as is evident in the various cultivars. The accumulated genetic variability provides an important resource of alleles to improve important ornamental traits; however, conventional breeding programs may not be sufficient to improve complex traits in chrysanthemum. Modern molecular breeding tools, such as molecular marker-assisted selection (MAS) breeding, could enhance important chrysanthemum ornamental traits. Hence, development of polymorphic markers is urgently needed.

Molecular markers are valuable tools used in genetic linkage map construction and MAS breeding. Among molecular markers, SSR markers are useful genetic markers because they are hypervariable, codominant, and highly informative. Further- more, compared with genomic SSRs, expressed sequence tag SSRs (EST-SSRs) will likely provide more possibility of finding associations with functional genes because they occur within the coding region and may affect gene function. EST-SSR markers also exhibit high transferability among closely related species and can be exploited as anchor markers for comparative mapping or evolutionary studies. However, EST-SSR markers have been developed for only a limited number of Chrysanthemum species (Feng et al., 2016; Han et al., 2018; Jo et al., 2015; Khaing et al., 2013; Li et al., 2013; Liu et al., 2015; Park et al., 2015; Wang et al., 2013; Zhang et al., 2014). As a result, anonymous dominant markers were predominantly used in earlier studies, e.g., earlier genetic maps of chrysanthemum were constructed using random amplified polymorphic DNA, intersimple sequence repeats, amplified fragment length polymorphism (Zhang et al., 2010), and sequence-related amplified polymorphism markers (Zhang et al., 2011). More recently, a single nucleotide polymorphism (SNP)-based map was constructed (Van Geest et al., 2017). However, because of the lack of available sequence information and the need for special instrumentations, SNP markers are difficult to use for most researchers. In addition, SSR markers are more informative than SNP markers, which are generally biallelic. Therefore, SSR marker technology remains important because of its simplicity and convenience combined with its sequencing-free assay, in particular for species that lack genome sequence data.

Here, we used the large dataset of EST sequences to identify EST-SSR markers for chrysanthemum. The specific aims of our study for chrysanthemum were as follows: 1) to characterize the EST-SSR loci and screen SSR primer polymorphisms in $F_{1}$ progeny, 2) to characterize the possible function of polymorphic SSR-containing unigenes, 3) to test cross-species/genus transferability within the genera Chrysanthemum and Ajania, and 4) to apply ESTSSR markers to phylogenetic relationships analysis.

\section{Materials and Methods}

Microsatellite identification, primer design, and polymorphism screening. A total of 88,499 ESTs, obtained from $C$. ×morifolium 'Jinbudiao' transcriptome sequences of flower buds, were submitted to the sequence read archive database at GenBank (www.ncbi.nlm.nih.gov), where they were combined and given the accession number SRP109613. These sequences were assembled further after redundancy elimination and produced 17,462 unigenes with a mean length of 1864 base pairs (bp). The MicroSAtellite program (MISA, http://pgrc.ipk-gatersleben.de/misa) was used to detect SSRs, and the search criteria for di-, tri-, tetra-, penta-, and hexanucleotide motifs were a minimum of six, five, five, four, and four repeats, respectively. Two or more SSRs 
separated by no more than $100 \mathrm{bp}$ were defined as compound SSRs. Mononucleotide and complex SSR types were excluded from the study. Compound ones that were separated by $0 \mathrm{bp}$ were considered. To predict the location of the SSR motifs in genes, the ORF Finder software (http://www.ncbi.nlm.nih.gov/gorf/gorf.html) was used to identify the initiation and termination codons in EST sequences. Primers were designed using Primer 3.0. A subset of 1584 primer pairs were selected for validation of marker assay performance.

Genomic DNA was isolated from fresh young leaves using a DNA extraction kit (Demeter Biotech, Beijing, China). DNA quality was checked on $1 \%$ agarose gels and quantified by Unico ultraviolet-visible Spectrophotometer (Unico, Franksville, WI). Polymerase chain reaction (PCR) was performed in $10 \mu \mathrm{L}$ of reaction mixture containing $25 \mathrm{ng}$ genomic DNA, $5 \mu \mathrm{L} 1 \times$ Power Taq PCR MasterMix (TsingKe, Beijing, China), $0.5 \mu \mathrm{L}$ of $10 \mu \mathrm{M}$ each primer, and $3 \mu \mathrm{L}$ sterile distilled water. The PCR reaction program was as follows: DNA denaturation at $94{ }^{\circ} \mathrm{C}$ for $3 \mathrm{~min}$; 35 cycles of $94{ }^{\circ} \mathrm{C}$ for $30 \mathrm{~s}$, the appropriate annealing temperature for $30 \mathrm{~s}$; $1 \mathrm{~min}$ at $72{ }^{\circ} \mathrm{C}$, and $72^{\circ} \mathrm{C}$ for $10 \mathrm{~min}$ as a final extension.

All EST-SSR primer pairs were initially screened using genomic DNA from the C. Xmorifolium 'Jinbudiao'. PCR products were separated by electrophoresis in $2 \%$ agarose gels. Generally, a subset of ESTSSR primers was identified as validated markers. All validated markers were screened for polymorphisms among the parents and six individuals of an $F_{1}$ mapping population. The use of closely related individuals in a population may enhance the reliability of our results. Amplified products that showed a band of the expected size were separated on $8 \%$ denaturing polyacrylamide gel electrophoresis and visualized by silver staining. The primers that were not successfully amplified or produced multiple bands were further tested by reducing or increasing the annealing temperature. The mapping population is maintained in the experimental fields at Xiaotangshan, which belongs to Beijing Forestry University, Beijing, China (lat. $40.0^{\circ} \mathrm{N}$, long. $\left.116.3^{\circ} \mathrm{E}\right)$.

Functional annotation. To identify the putative function of SSR-containing unigenes, the unigenes harboring polymorphic microsatellite were subjected to BLASTX search against the National Center for Biotechnology Information nonredundant protein database, the Gene Ontology (GO) database, the Cluster of Orthologous Groups (COG) database, and the Kyoto Encyclopedia of Genes and Genomes (KEGG) database. Sequences with $\mathrm{E}$ value $<10^{-6}$ and scores $>50$ were annotated.

Cross-species/genera transferability and evaluation of genetic relationships within 59 samples. To assess cross-species/genera transferability, 25 randomly selected polymorphic EST-SSRs were genotyped in 59 samples, including 36 Chrysanthemum, 10 Ajania, and 13 intergeneric hybrids (Supplemental Table 1). The PCR amplification reaction system was conducted as mentioned previously. PCR product amplification was directly separated on $8 \%$ denaturing polyacrylamide gel electrophoresis and visualized by silver staining. All reliable bands were scored as 1, 0 for presence and absence, and transformed into a binary matrix. expected heterozygosities $\left(H_{\mathrm{o}}\right.$ and $H_{\mathrm{e}}$, respectively), Shannon index (I), and PIC were calculated for each marker using an $\mathrm{R}$ package (an R package that is suitable for hexaploids).

The discriminatory abilities of EST-SSR markers were estimated using cluster analyses to assess genetic relationships among 59 samples. The binary data were analyzed using the Numerical Taxonomy Multivariate Analysis System (NTSYS-pc) version 2.10 software with SIMQUAL module to calculate genetic similarity (GS) coefficients based on coefficient for similarity matching (Rohlf, 1998). A neighbor-joining (NJ) dendrogram was constructed using the GS matrix by the unweighted pair group method with arithmetic average (UPGMA). Bootstrapping analysis (1000 resamplings) was carried out using software FREETREE V0.9.1.50 (Pavlicek et al., 1999). Bootstrap values more than 50 are considered significant and provided on the dendrogram. Biplot was computed based on the PCA of molecular data using software MVSP v3.1.3. The data were also analyzed using PCA to further demonstrate the multiple dimensional distributions of the 59 samples in a scatter-plot.

Population structure was investigated using a model-based approach, which is implemented in the software package STRUCTURE 2.3.4. Different population genetic clusters $(\mathrm{K}=1-10)$ were evaluated with five runs per $\mathrm{K}$ value. For each run, the initial burn-in period was set to 100,000 with 2000,000 MCMC iterations, under the admixture model and independent allele frequencies, with no prior information on the origin of individuals. To determine the most probable value of $\mathrm{K}$, the deltaK method described by Evanno et al. (2005) was used as implemented in Microsoft Excel 2016. STRUCTURE results were displayed in the program STRUCTURE PLOT (Ramasamy et al., 2014).

\section{Results}

Characteristics of EST-SSR markers in chrysanthemum transcriptome. By sequencThe number of alleles $\left(N_{a}\right)$, observed and

ing the transcriptome of chrysanthemum, we identified 4661 EST-SSR loci located in 3823 $(21.9 \%)$ unigene sequences from $3.26 \mathrm{Mb}$ total sequence, with an average of 1 SSR per $6.98 \mathrm{~kb}$ (Table 1). The SSRs were not evenly distributed, with 688 (3.9\%) being unigenes containing more than one SSR locus (Table 1). Analysis of all 4661 EST-SSR loci showed that trinucleotide $(30.04 \%, 1400)$ and dinucleotide repeat motifs $(17.89 \%, 834)$ predominated, followed by tetranucleotide $(1.57 \%$, $73)$, pentanucleotide $(0.13 \%, 6)$, and hexanucleotide repeat motifs $(0.09 \%$, 4) (Table 1$)$.

The frequency distribution of 35 major types with di- and trinucleotide repeat motifs and repeat number of EST-SSRs are shown in Fig. 1. Of the identified SSRs, the dinucleotide repeat AC/GT $(31.29 \%$, 261) was the most plentiful motif, followed by $\mathrm{CA} / \mathrm{TG}$ $(27.82 \%, 232), \mathrm{AT} / \mathrm{TA}(17.27 \%, 144), \mathrm{TCA}$ TGA $(10.2 \%, 143)$, ATC/GAT $(9.3 \%, 130)$, and $\mathrm{AG} / \mathrm{CT}(12.35 \%, 103)$ (Fig. $1 \mathrm{~A}$ and $\mathrm{B}$ ). SSR length was mostly 12 to $20 \mathrm{bp}$, accounting for $93.7 \%$ of the SSRs. The largest microsatellite identified was $78 \mathrm{bp}$, with a hexanucleotide motif repeated 13 times (Fig. 1C).

SSR primers screening and polymorphism testing. Using $C$. ×morifolium 'Jinbudiao', $831(52.5 \%)$ primer pairs were successfully amplified (Supplemental Table 2). Moreover, 180 primer pairs amplified bands larger than expected, or produced multiple bands. The remaining 573 primer pairs did not generate PCR products after subjecting them to various PCR programs.

These 831 primer pairs were further subjected to screening for polymorphisms in the $\mathrm{F}_{1}$ progeny, including the parents and six individuals. A total of 361 primer pairs (43.4\%) amplified reproducible polymorphic bands, including 95 di-, 257 tri-, 8 tetra-, and 1 compound motif loci. A total of 1396 alleles were detected, and the number of alleles per locus ranged from 2 to 8 , with an average of 3.87 (Supplemental Table 3).

We also investigated the distribution of microsatellites in the transcript (coding sequence [CDS], 5' untranslated region [UTR], and $3^{\prime}$ UTR). Of the 831 validated SSR markers, 400 (48\%), 340 (41\%), and 91 $(11 \%)$ were located in the CDS, $5^{\prime} \mathrm{UTR}$, and $3^{\prime}$ UTR, respectively. In addition, 96\% of direpeats and $100 \%$ of tetrarepeats were located within the UTR, whereas $67 \%$ of the trirepeats occurred within the CDS.

Table 1. Summary of expressed sequence tag-simple sequence repeat (EST-SSR) markers identified in the chrysanthemum transcriptome.

\begin{tabular}{lc}
\hline Searching items & Numbers \\
\hline Total number of sequences examined & 17,462 \\
Total size of examined sequences $(\mathrm{Mb})$ & 3.26 \\
Total number of identified SSRs & 4,661 \\
Number of SSR-containing sequences & 3,823 \\
Frequency of SSRs & $1 / 6.98 \mathrm{~kb}$ \\
Number of sequences containing more than 1 SSR & 688 \\
Number of SSRs present in compound formation & 305 \\
Dinucleotide & 834 \\
Trinucleotide & 1,400 \\
Tetranucleotide & 73 \\
Pentanucleotide & 6 \\
Hexanucleotide & 4
\end{tabular}


A

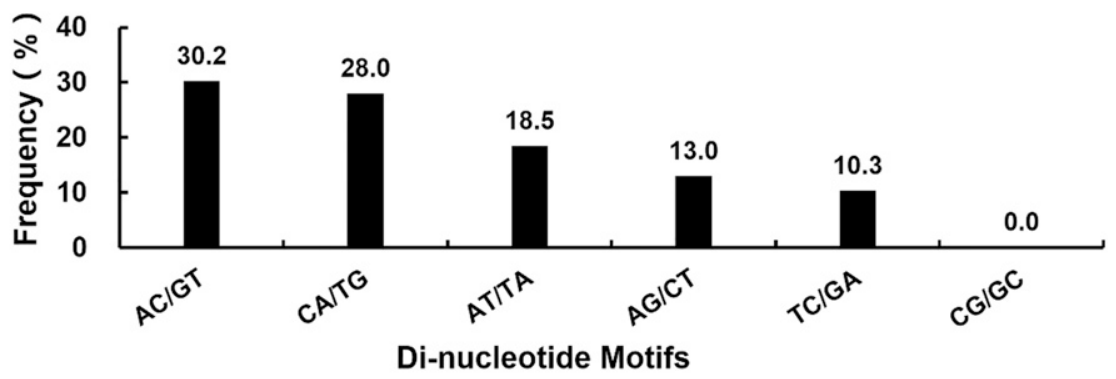

B

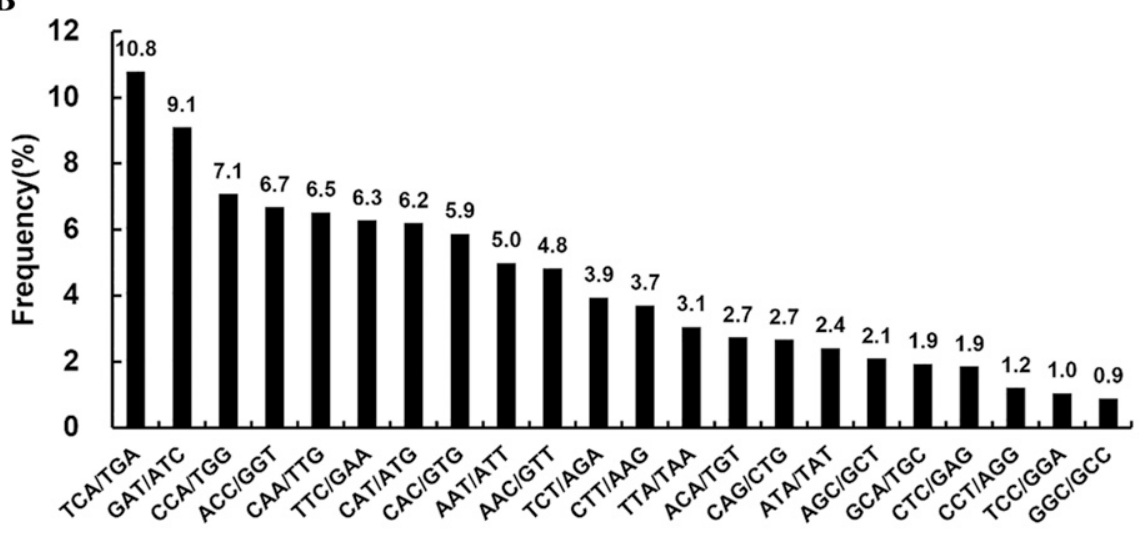

Tri-nucleotide Motifs

C

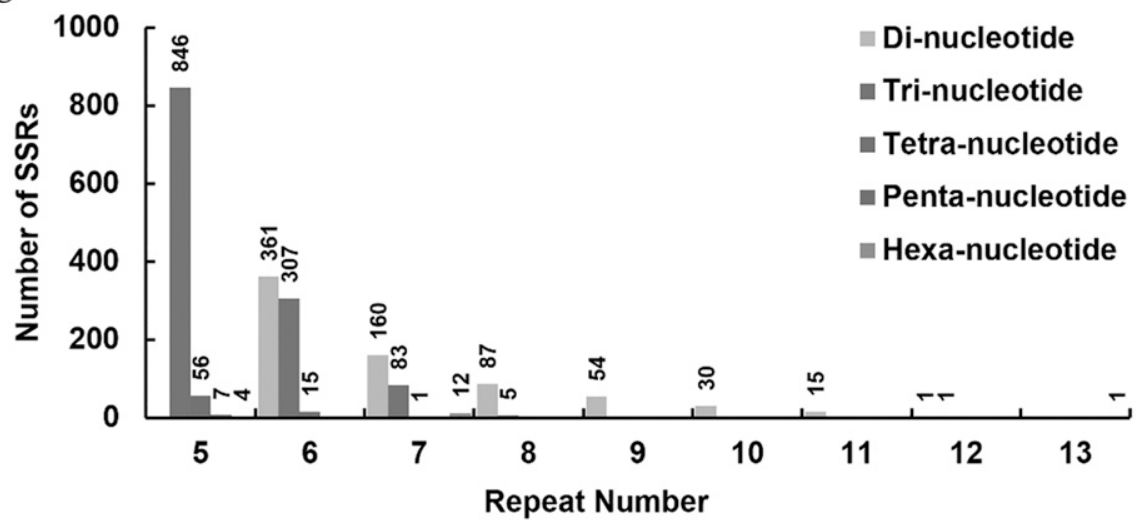

Fig. 1. Characterization of sample sequence repeats (SSRs) in chrysanthemum transcriptome. (A) Frequency distribution of dinucleotide SSRs based on motif type. (B) Frequency distribution of trinucleotide SSRs based on main motif type. (C) Number of different repeat motifs.

Functional annotation. Of the 361 polymorphic SSR-containing unigenes, 330 (93.5\%) possessed sequence similarities and could be assigned putative functions (Supplemental Table $3)$. Several unigenes encoded proteins similar to important transcription factors, such as CEM190-containing unigenes encode proteins similar to transcription factor APETALA2 (AP2), CEM505-containing unigenes encode proteins similar to transcription factor $b H L H$, and CEM512-containing unigenes encode proteins similar to zinc finger protein CONSTANS (CO). In addition, 246, 83, and 71 SSR-containing unigenes were assigned to GO annotations, COG classifications, and the KEGG database, respectively (Supplemental Fig. 1A-C). Annotation with GO confirmed that the highest proportion of unigenes were related to cell, cell part, and metabolic process. COG classifi- cation of the unigenes revealed that the most highly represented functional group was "general function," followed by "replication, recombination, and repair." KEGG pathway enrichment analysis showed that the pathways with the most abundant unigenes are spliceosome and messenger RNA surveillance pathway. However, the exact function for these SSR-containing unigenes needs further research.

Cross-species transferability of 25 polymorphic EST-SSR markers. To assess the utility of the EST-SSR markers beyond $C$. $\times$ morifolium, we selected 25 markers to evaluate whether they were amplifiable in other related species/ genera, including Chrysanthemum and Ajania species/genera (Supplemental Table 1, codes 1-12). All the EST-SSR primers demonstrated successful cross-species amplification (Sup- plemental Table 4). Of these, 16 (64\%) were amplified in all species. Transferability of the primers among Chrysanthemum species was identified in C. indicum (ind1) (100\%), Chrysanthemum mankingense (100\%), Chrysanthemum chanetii (96\%), C. indicum (ind2) (96\%), C. zawadskii (96\%), and C. indicum var. aromaticum (92\%). In Ajania, the rate of amplification was slightly lower, and transferability was present with Ajania potaninii (100\%), Ajania khartensisc (92\%), Ajania fruticulosa (92\%), Ajania achilloides (88\%), Ajania purpurea (88\%), and Ajania scharnhorstii $(76 \%)$. These data suggest that the EST-SSR markers developed in this study could be efficiently applied to the genus Chrysanthemum and Ajania.

Evaluation of genetic relationships within 59 samples. The 25 representative EST-SSR markers also were selected to estimate their polymorphism in 59 samples. The allelic variation in these samples was assessed to determine whether these EST-SSR markers could be effectively used to identify genetic variability. In total, 151 alleles were detected with an average of 6 alleles per marker, ranging from 2 at CEM081 to 13 at CEM814 (Table 2). The PIC values ranged from 0.29 for CEM081 to 0.86 for CEM814, with a mean value of 0.67 . The other parameters $\left(N_{a}, H_{o}, H_{e}\right.$, Shannon index) are shown in Table 2.

In additional analysis, we tested the discriminatory capacity by determining whether the 25 markers were able to recapitulate the known phylogenetic relationships among the 59 samples. The NJ tree (Fig. 2A), based on the shared allele coefficients, clustered them into six main groups. Cluster I comprised three species (ind1, cha, ind2) and cluster II contained nine species, of which three species (man, ind3, zaw) belong to Chrysanthemum and six species (fru, pot, ach, sch, kha, pur) belong to Ajania. The relationships between cluster I and cluster II are very close. Cluster III included four samples (pac1, 2, 3, 4) belonging to Ajania pacifica. There are eight samples (chr1, 2, 3, 4, 5, 6, 7, 8) in cluster IV, which are all cultivars of large chrysanthemum. Cluster V consisted of 13 intergeneric hybrids of the $C$. ×morifolium 'Pudidanfen' (male parent, hexaploid) and $A$. pacifica (female parent, decaploid), and cluster VI was composed of 22 samples, including groundcover chrysanthemum and escarpment chrysanthemum.

Population structure estimation for the 59 samples was done using the Bayesian clustering approach implemented in STRUCTURE 2.3.4. Given that the log likelihood values increased progressively as $\mathrm{K}$ increased, the method of Evanno et al. (2005) was applied as a criterion to infer the most likely $\mathrm{K}$ value. The maximum delta $\mathrm{K}$ was detected at $\mathrm{K}=6$, inferring that all 59 individuals can be grouped into six groups with the highest probability (Fig. 2B). Cluster A was composed of man, ind2, ind3, zaw, fru, pot, ach, sch, kha, pur, and ind1; cluster $\mathrm{B}$ was composed of cha, chr1, chr2, and sm; cluster C was the same as cluster V in the NJ tree; cluster D was the same as cluster III and was composed of pac1, 2, 3, and 4; cluster E consisted of chr3, 4, 5, 6, 7, 
Table 2. Polymorphism information of 25 expressed sequence tag-simple sequence repeat (EST-SSR) markers in 59 chrysanthemum samples, including the following parameters: the number of alleles $\left(N_{\mathrm{a}}\right)$, observed and expected heterozygosities $\left(H_{\mathrm{o}}\right.$ and $\left.H_{\mathrm{e}}\right)$, PIC, and Shannon index.

\begin{tabular}{lccccc}
\hline Primer name & $N_{\mathrm{a}}$ & $H_{\mathrm{o}}$ & $H_{\mathrm{e}}$ & PIC & Shannon index \\
\hline CEM076 & 6 & 0.92 & 0.69 & 0.64 & 1.31 \\
CEM080 & 6 & 0.88 & 0.79 & 0.75 & 1.63 \\
CEM081 & 2 & 0.29 & 0.35 & 0.29 & 0.54 \\
CEM082 & 5 & 0.59 & 0.75 & 0.70 & 1.43 \\
CEM088 & 8 & 0.69 & 0.80 & 0.77 & 1.75 \\
CEM091 & 4 & 0.90 & 0.70 & 0.65 & 1.27 \\
CEM093 & 3 & 0.48 & 0.62 & 0.55 & 1.03 \\
CEM371 & 8 & 0.85 & 0.75 & 0.71 & 1.57 \\
CEM372 & 6 & 0.88 & 0.63 & 0.55 & 1.18 \\
CEM380 & 7 & 0.73 & 0.83 & 0.81 & 1.86 \\
CEM496 & 10 & 0.81 & 0.80 & 0.77 & 1.83 \\
CEM501 & 5 & 0.95 & 0.75 & 0.71 & 1.49 \\
CEM504 & 4 & 0.71 & 0.70 & 0.64 & 1.28 \\
CEM505 & 5 & 0.93 & 0.79 & 0.76 & 1.59 \\
CEM508 & 0.54 & 0.70 & 0.64 & 1.30 \\
CEM512 & 5 & 0.73 & 0.62 & 0.55 & 1.14 \\
CEM513 & 5 & 0.91 & 0.83 & 0.80 & 1.87 \\
CEM514 & 8 & 0.65 & 0.57 & 1.11 \\
CEM515 & 6 & 0.74 & 0.70 & 1.51 \\
CEM519 & 4 & 0.88 & 0.74 & 0.70 & 1.46 \\
CEM685 & 7 & 0.78 & 0.85 & 0.83 & 1.92 \\
CEM723 & 5 & 0.76 & 0.66 & 0.59 & 1.17 \\
CEM780 & 7 & 0.66 & 0.78 & 0.76 & 1.75 \\
CEM782 & 4 & 0.46 & 0.88 & 0.89 \\
CEM814 & 8 & 0.92 & 0.86 & 2.26 \\
Mean & 6 & & 0.67 & 1.45 \\
\hline
\end{tabular}

and 8; and cluster $\mathrm{F}$ was composed of 21 cultivars. The results were quite similar to the NJ tree, with the exception of chr1, 2, and sm.

The genotyping data were also subjected to PCA analysis to obtain an alternative perspective of the phylogenetic relationships among the 59 samples. PCA showed similar results with the NJ tree, except that the major groups I and II formed one cluster in the twodimensional plot (Fig. 2C).

\section{Discussion}

Next-generation sequencing technology provides a simple and effective way to develop molecular markers such as SSRs and SNPs, of which few are available for chrysanthemum. Before this research, only 7300 Chrysanthemum ESTs (http://www.ncbi. nlm.nih.gov/genbank/dbest/dbest_summary/) had been generated, and Feng et al. (2016) developed 17 novel EST-SSR markers from them. The EST-SSRs developed in the current study will prove directly useful for chrysanthemum, and readily transferable to closely related species, as illustrated here between Chrysanthemum and Ajania.

In the chrysanthemum transcriptome, the mean length of the unigenes was $1864 \mathrm{bp}$, which was much longer than reported in previous transcriptome studies of the tree peony (mean length $698 \mathrm{bp}$ ) (Wu et al., 2014) and Neolitsea sericea (mean length 733 bp) (Chen et al., 2015). It revealed that the overall sequence data from $C$. ×morifolium 'Jinbudiao' were effectively assembled to create longer DNA sequences or contigs. Longer sequences provide longer lengths for selection of SSR flanking sequence, which have been found to assist in designing SSR primers (Zalapa et al., 2012). A survey on dicotyledonous species reported that the proportion of ESTs containing SSRs ranged from $2.65 \%$ to $16.82 \%$ (Varshney et al., 2005). In this study, $\approx 21.9 \%$ of 17,462 ESTs contained at least one SSR, which was higher than the upper limit of the range, as well as other reported values, such as Stylosanthes guianensis $(9.96 \%)$ (Ding et al., 2015). Furthermore, the distribution density of EST-SSRs was one SSR in every $6.98 \mathrm{~kb}$. This value was higher than the frequency reported in the tree peony $(1 / 9.24 \mathrm{~kb})$ (Wu et al., 2014) and Pinus dabeshanensis $(1 / 23.08 \mathrm{~kb})$ (Xiang et al., 2015), but lower than that in N. sericea (1/ $3.8 \mathrm{~kb}$ ) (Chen et al., 2015) and taro (1/5.90 kb) (You et al., 2015). Chrysanthemum may have a higher frequency of EST-SSRs, but alternative explanations, such as the size of the data set, SSR search criteria, and database-mining tools also should be considered (Varshney et al., 2005).

Among the various sequence repeats, trinucleotides were predominant (Table 1), consistent with studies in plants such as $C$. indicum (Han et al., 2018), rice (Lawson and Zhang, 2006), Brassica rapa (Zheng et al., 2016), and Helianthus annuus (Pramod et al., 2014). This observation is common for EST-derived SSRs, as changes in trinucleotide repeat number will not cause frameshift mutaSSRs with AC/GT motifs (31.29\%) were the most frequent (Fig. 1A), which agrees with results reported in Chrysanthemum nankingense (Wang et al., 2013) and C. indicum (Han et al., 2018). The most prevalent trinucleotide motif type often differs among species. In chrysanthemum, TCA (10.2\%) predominated, unlike those in Dysosma versipellis, S. guianensis, and N. sericea (AAG) tions. Our analysis of EST-SSRs found that
(Chen et al., 2015; Ding et al., 2015; Guo et al., 2014); taro (CCG) (You et al., 2015); P. dabeshanensis (AGC) (Xiang et al., 2015); and tree peony (CCA) (Wu et al., 2014). In contrast, $\mathrm{CCG} / \mathrm{CGG}(0.6 \%)$ motifs were very rare (see Fig. 1), found mostly in monocots such as maize, barley, and taro (Morgante et al., 2002; Toth et al., 2000; You et al., 2015). The observed rarity of CCG/CGG repeat units has been reported in some dicots, such as the common bean, $D$. versipellis, $S$. guianensis, $N$. sericea, $P$. dabeshanensis, and the tree peony (Chen et al., 2014, 2015; Ding et al., 2015; Guo et al., 2014; Wu et al., 2014; Xiang et al., 2015). The extremely low number of SSR motifs containing $\mathrm{C}$ and $\mathrm{G}$ could be attributed to the composition of dicot genes being less rich in $\mathrm{G}+\mathrm{C}$.

Our results indicated that EST-SSRs in the chrysanthemum transcriptome do not evenly distribute across CDS and UTR regions, with a greater frequency in the latter. The result was consistent with that of other transcriptome surveys (Liu et al., 2016; Pramod et al., 2014). The density of microsatellites in the chrysanthemum unigene database may be dependent on two factors: 1) evolutionary constraints due to the harmful effect of microsatellite mutation on gene function, and 2) the directional selection on microsatellites with adaptive roles (Liu et al., 2016). As microsatellites are highly prone to indel mutations by means of slip-strand mispairing (Levinson and Gutman, 1987), it seems that microsatellites in the CDS regions are more inclined to obstruct gene function, leading to higher evolutionary constraints on microsatellites in the CDS region. Besides, microsatellites in the UTRs may confer evolutionarily adaptive roles on genes with regard to tuning ability. Thus, the directional selection may favor microsatellite expansion in UTRs.

In the current study, $52.5 \%$ of the ESTSSR primers amplified the target sequence, which is lower than the success rate of $60 \%$ to $90 \%$ amplification reported in previous studies, such as Pinus dabeshanensis $(81.71 \%)$, Stylosanthes (83.34\%), sweetpotato $(84.6 \%)$, and pigeon pea (71.24\%) (Ding et al., 2015; Dutta et al., 2011; Wang et al., 2011; Xiang et al., 2015), but higher than in tree peony (47.3\%), Dysosma versipellis (42.5\%), and Neolitsea sericea (16\%) (Chen et al., 2015; Guo et al., 2014; Wu et al., 2014). The success ratio may result from primer design, selecting the best performing loci from the total number of sequences available is a key component in the successful development of SSR markers (Zalapa et al., 2012).

Of the 831 valid EST-SSR markers, 361 (43.4\%) polymorphic markers were obtained, thereby providing powerful tools for constructing an SSR-based linkage map in chrysanthemum. Research showed that when SSRs $\geq 20 \mathrm{bp}$ tend to be hypervariable markers, $12 \mathrm{bp} \leq$ SSRs $<20$ bp tend to be potentially variable markers (Temnykh et al., 2001). The lengths of SSRs in this study were mostly 12 to 20 bp (93.7\%), which could guarantee a medium degree of polymorphism. 

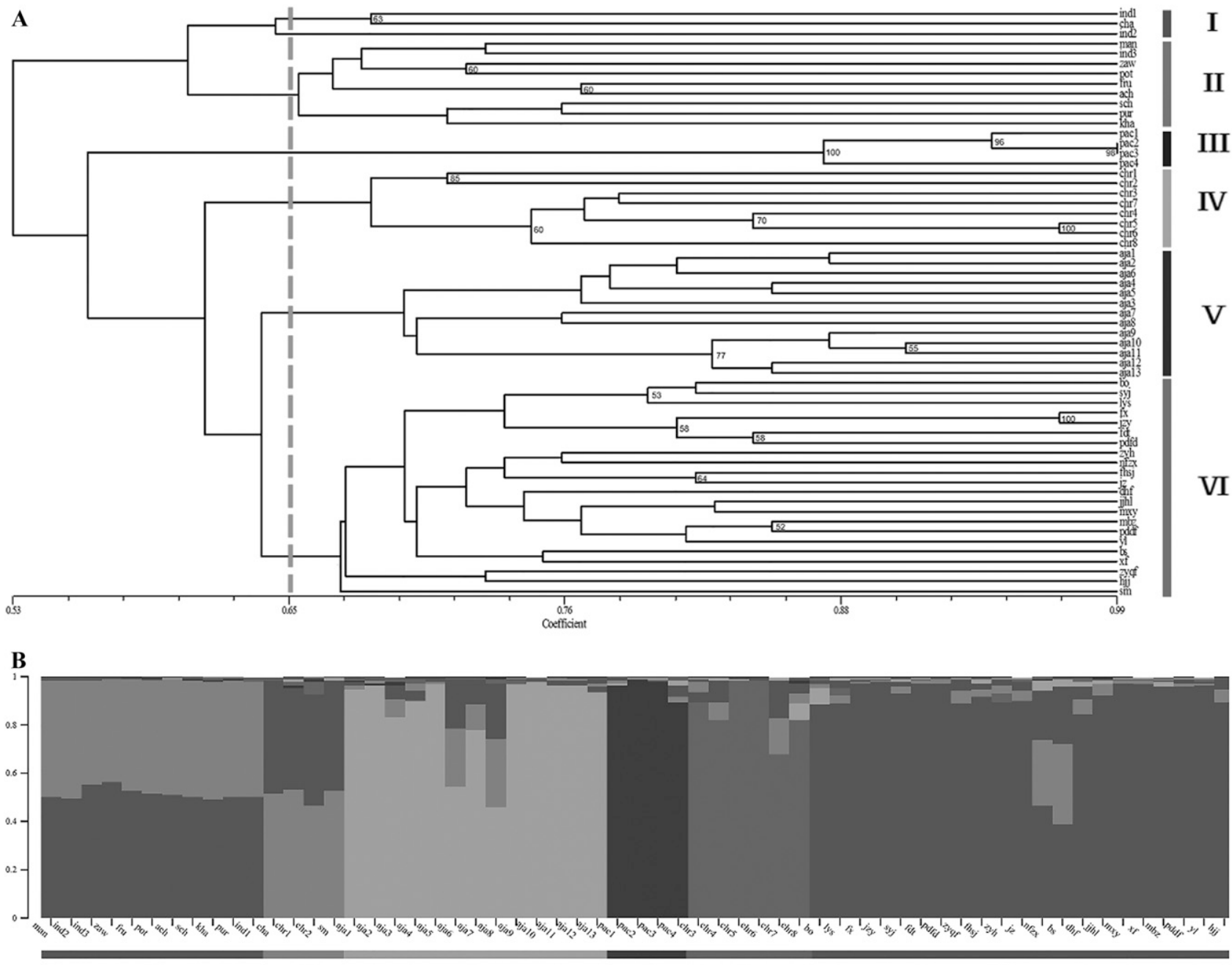

A

B

C

D

E

F

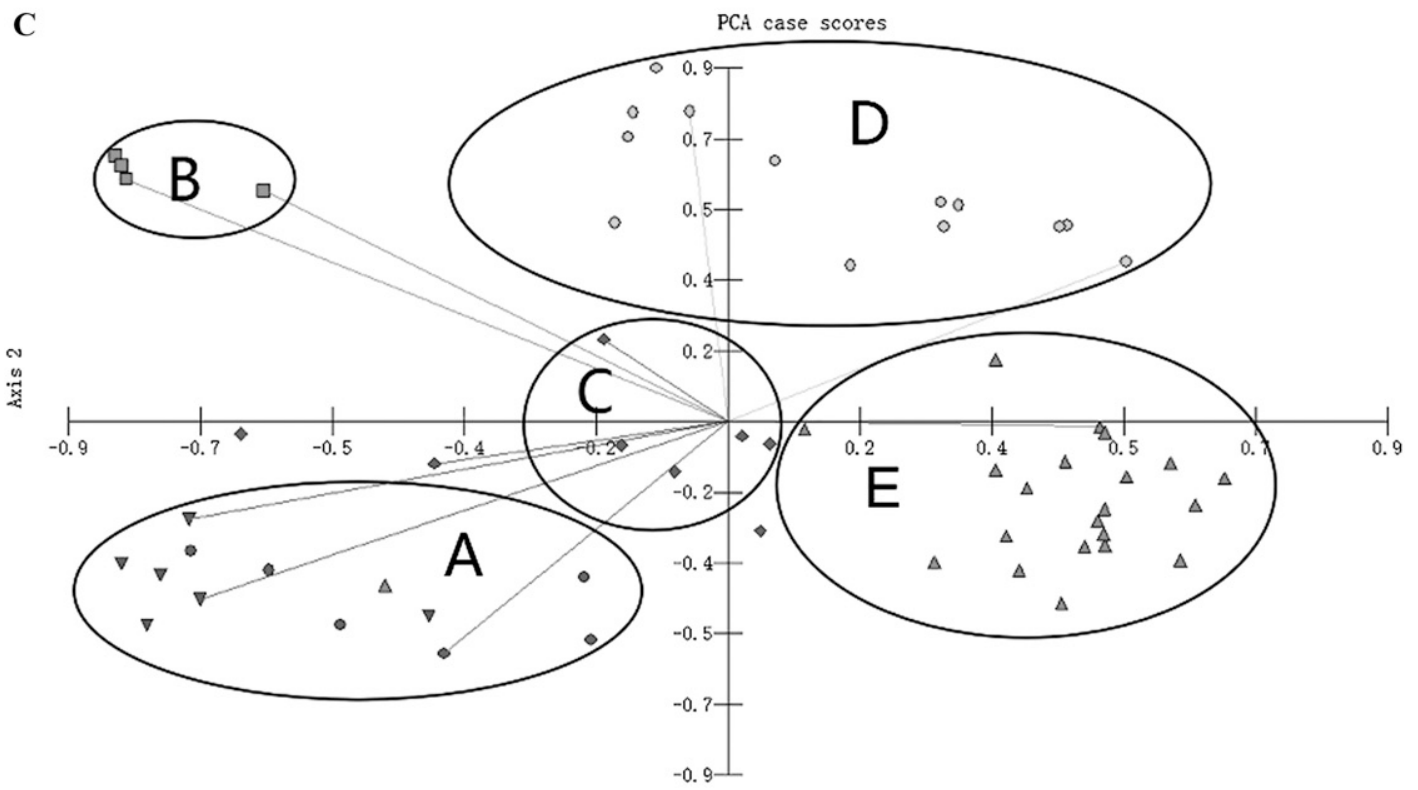

- Chrysanthemum

$\nabla$ Ajania

A. pacifica

Large chrysanthemoum

- intergeneric hybrids

$\Delta$ cultivars

Axis 1

Fig. 2. (A) Genetic relationships among 59 samples based on expressed sequence tag-simple sequence repeat (EST-SSR) markers. The dendrogram shows the genetic relationships among 59 samples. The scale at the bottom of the dendrogram indicates the level of similarity between the genotypes, bootstrap values ( $>50)$ were labeled on the branches from 1000 resamplings. The information of the abbreviations represented in the tree can be seen in Supplemental Table 1. (B) STRUCTURE analysis at $\mathrm{K}=6$ for 59 samples. The colors represent different clusters and the colors in each accession represent the average proportion of alleles that placed each accession under two or more clusters. The text below the figure refers to accession names. (C) Two-dimensional plot of principal component analysis (PCA) of 59 samples. The circles indicate the group of samples that are similar to each other in the PCA analysis (A, B, C, D, and E are the five major clusters). 
Moreover, the number of samples and the geographic origin of samples used may have led to a different ratio of polymorphisms. If more samples were screened, the number of polymorphic SSRs in this study would most likely increase.

SSRs are usually considered as "junk DNA" or evolutionarily neutral DNA markers in the genome. Recently, microsatellites have increasingly been identified and characterized within protein-coding genes and their UTRs, providing numerous lines of evidence for the potential functions of microsatellites. When microsatellites locate in a functional gene, their repeat motif variation can influence gene regulation, transcription, translation, and protein function. In the current study, a number of polymorphic SSR-containing unigenes have transcription factor activity or other functions. A similar investigation of the function of microsatellite in Arabidopsis (Lawson and Zhang, 2006) and Sargassum thunbergii (Liu et al., 2016) showed that some transcripts harboring microsatellites were also related to the transcription factors. In Elaeis guineensis (Tranbarger et al., 2012), microsatellite polymorphisms were also found in sequences encoding $A P 2$-like, $b Z I P$, zinc finger, $M A D S$ box, and NAC-like transcription factors, as well as other transcriptional regulatory proteins and several RNA interacting proteins. SSRs in transcripts encoding proteins related to transcription and other functions may provide pertinent markers for MAS.

The PIC values were used to estimate the informativeness of the markers, which was categorized as high (PIC > 0.5), moderate $(0.5<$ PIC $<0.25)$, and low $(\mathrm{PIC}<0.25)$, respectively (Botstein et al., 1980). In the present research, 23 of 25 primers exhibited high PIC values, the mean PIC value of 0.67 was lower compared with a previous report for medicinal Chrysanthemum cultivars (0.97) (Feng et al., 2016), but higher than Phaseolus vulgaris (0.47), Ipomoea nil $(0.41 \pm 0.14)$, and faba bean (0.19) (ElRodeny et al., 2014; Garcia et al., 2011; Ly et al., 2012). Both UPGMA method and STRUCTURE analyses revealed that the 59 samples were divided into six clusters (Fig. 2A and B). Our results indicated that samples of Chrysanthemum had a closer genetic relationship with those of Ajania, which is in agreement with previous findings that Ajania should be combined into the genus of Chrysanthemum (Luo et al., 2018; Ohashi and Yonekura, 2004; Zhao et al., 2009). There was significant genetic differentiation between wild species and cultivars in Chrysanthemum. In the case of cultivars, eight large chrysanthemums were clustered together and are closely related to A. pacifica, indicating that large chrysanthemums may have a higher ploidy. And the intergeneric hybrids were grouped together (Liu et al., 2015). These results suggest there is substantial differentiation in the genetic relationships between different genera and various cultivars. The incongruence between the dendrogram and the division of cultivars may be attributed to a recording error during the data-collecting process.
In conclusion, 831 valid and 361 polymorphic EST-SSR markers have been developed for chrysanthemum, substantially increasing the molecular marker repository for chrysanthemum. The transferability analysis and phylogenetic relationships analysis have validated the universality of the 25 randomly selected polymorphic EST-SSRs. They were transferable to Ajania species and can distinguish the phylogenetic relationships between wild species and cultivars. Some SSR-containing sequences were assigned putative functions, which will require further study. These EST-SSR markers provide a valuable genomic resource for cultivar identification and characterization, genetic diversity assessment, genetic linkage mapping, and MAS in Chrysanthemum species and related taxa.

\section{Literature Cited}

Botstein, D., R.L. White, M.H. Skolnick, and R.W. Davies. 1980. Construction of a genetic linkage map in man using restriction fragment length polymorphisms. Amer. J. Hum. Genet. 32:314 331.

Chen, L., Y. Cao, N. Yuan, K. Nakamura, G. Wang, and Y. Qiu. 2015. Characterization of transcriptome and development of novel ESTSSR makers based on next-generation sequencing technology in Neolitsea sericea (Lauraceae) endemic to East Asian land-bridge islands. Mol. Breed. 35(9):187.

Chen, M., J. Wu, L. Wang, X. Zhang, M.W. Blair, J. Jia, and S. Wang. 2014. Development of mapped simple sequence repeat markers from common bean (Phaseolus vulgaris L.) based on genome sequences of a Chinese landrace and diversity evaluation. Mol. Breed. 33:489-496.

Dai, S.L., J.Y. Chen, and W.B. Li. 1998. Application of RAPD analysis in the study on the origin of Chinese cultivated chrysanthemum. Acta Bot. Sin. 11:1053-1059.

Ding, X., Q. Jia, X. Luo, L. Zhang, H. Cong, G. Liu, and C. Bai. 2015. Development and characterization of expressed sequence tag-derived simple sequence repeat markers in tropical forage legume Stylosanthes guianensis (Aubl.) Sw. Mol. Breed. 35:202.

Dutta, S., G. Kumawat, B.P. Singh, D.K. Gupta, S. Singh, V. Dogra, K. Gaikwad, T.R. Sharma, R.S. Raje, T.K. Bandhopadhya, S. Datta, M.N. Singh, F. Bashasab, P. Kulwal, K.B. Wanjari, R.K. Varshney, D.R. Cook, and N.K.N.N Singh. 2011. Development of genic-SSR markers by deep transcriptome sequencing in pigeonpea [Cajanus cajan (L.) Millspaugh]. BMC Plant Biol. 11:17.

El-Rodeny, W., M. Kimura, H. Hirakawa, A. Sabah, K. Shirasawa, S. Sato, S. Tabata, S. Sasamoto, A. Watanabe, K. Kawashima, M. Kato, T. Wada, H. Tsuruoka, C. Takahashi, C. Minami, K. Nanri, S. Nakayama, M. Kohara, M. Yamada, Y. Kishida, T. Fujishiro, and S. Isobe. 2014. Development of EST-SSR markers and construction of a linkage map in faba bean (Vicia faba). Breed. Sci. 64:252-263.

Evanno, G., S. Regnaut, and J. Goudet. 2005. Detecting the number of clusters of individuals using the software structure: A simulation study. Mol. Ecol. 14:2611-2620.

Feng, S., R. He, J. Lu, M. Jiang, X. Shen, Y. Jiang, Z. Wang, and H. Wang. 2016. Development of SSR markers and assessment of genetic diversity in medicinal Chrysanthemum morifolium cultivars. Front. Genet. 7:113.
Garcia, R.A., P.N. Rangel, C. Brondani, W.S. Martins, L.C. Melo, M.S. Carneiro, T.C. Borba, and R.P. Brondani. 2011. The characterization of a new set of EST-derived simple sequence repeat (SSR) markers as a resource for the genetic analysis of Phaseolus vulgaris. BMC Genet. 12:41.

Guo, R., Y. Mao, J. Cai, J. Wang, J. Wu, and Y. Qiu. 2014. Characterization and cross-species transferability of EST-SSR markers developed from the transcriptome of Dysosma versipellis (Berberidaceae) and their application to population genetic studies. Mol. Breed. 34:17331746.

Han, Z., X. Ma, M. Wei, T. Zhao, R. Zhan, and W. Chen. 2018. SSR marker development and intraspecific genetic divergence exploration of Chrysanthemum indicum based on transcriptome analysis. BMC Genomics 19:291.

Jo, K., Y. Jo, H. Chu, S. Lian, and W.K. Cho. 2015. Development of EST-derived SSR markers using next-generation sequencing to reveal the genetic diversity of 50 chrysanthemum cultivars. Biochem. Syst. Ecol. 60:37-45.

Khaing, A.A., K.T. Moe, W.J. Hong, C.S. Park, K.H. Yeon, H.S. Park, D.C. Kim, B.J. Choi, J.Y. Jung, S.C. Chae, K.M. Lee, and Y.J. Park. 2013. Phylogenetic relationships of chrysanthemums in Korea based on novel SSR markers. Genet. Mol. Res. 12:5335-5347.

Klie, M., S. Schie, M. Linde, and T. Debener. 2014 The type of ploidy of chrysanthemum is not black or white: A comparison of a molecular approach to published cytological methods. Front. Plant Sci. 5:479.

Lawson, M.J. and L. Zhang. 2006. Distinct patterns of SSR distribution in the Arabidopsis thaliana and rice genomes. Genome Biol. 7:R14.

Levinson, G. and G.A. Gutman. 1987. Slippedstrand mispairing: A major mechanism for DNA sequence evolution. Mol. Biol. Evol. 4:203-221.

Li, Y.H., C. Luo, Z.Y. Wu, X.H. Zhang, X. Cheng, R. Dong, and C.L. Huang. 2013. Microsatellite enrichment by magnetic beads in chrysanthemum. Acta Hort. 977:269-278.

Liu, F., Z. Hu, W. Liu, J. Li, W. Wang, Z. Liang, F. Wang, and X. Sun. 2016. Distribution, function and evolution characterization of microsatellite in Sargassum thunbergii (Fucales, Phaeophyta) transcriptome and their application in marker development. Sci. Rep. 6:18947.

Liu, H., Q.X. Zhang, M. Sun, H.T. Pan, and Z.X. Kong. 2015. Development of expressed sequence tag-simple sequence repeat markers for Chrysanthemum morifolium and closely related species. Genet. Mol. Res. 14:7578 7586.

Luo, C., D. Chen, X. Cheng, H. Liu, Y. Li, and C. Huang. 2018. SSR analysis of genetic relationship and classification in Chrysanthemum germplasm collection. Hort. Plant J. 4:73-82.

Ly, T., H. Fukuoka, A. Otaka, A. Hoshino, S. Iida, E. Nitasaka, N. Watanabe, and T. Kuboyama. 2012. Development of EST-SSR markers of Ipomoea nil. Breed. Sci. 62:99-104.

Morgante, M., M. Hanafeyand, and W. Powell. 2002. Microsatellites are preferentially associated with nonrepetitive DNA in plant genomes. Nat. Genet. 30:194-200.

Ohashi, H. and K. Yonekura. 2004. New combinations in Chrysanthemum (Compositae-Anthemideae) of Asia with a list of Japanese species. J. Jpn. Bot. 79:186-195.

Park, S.K., P. Arens, D. Esselink, J.H. Lim, and H.K. Shin. 2015. Analysis of inheritance mode in chrysanthemum using EST-derived SSR markers. Scientia Hort. 192:80-88. 
Pavlicek, A., S. Hrda, and J. Flegr. 1999. FreeTree-freeware program for construction of phylogenetic trees on the basis of distance data and bootstrap/jackknife analysis of the tree robustness. Application in the RAPD analysis of genus Frenkelia. Folia Biol-Prague 45:9799.

Pramod, S., A.D. Perkinsand, and M.E. Welch. 2014. Patterns of microsatellite evolution inferred from the Helianthus annuus (Asteraceae) transcriptome. J. Genet. 93:431-442.

Ramasamy, R.K., S. Ramasamy, B.B. Bindroo, and V.G. Naik. 2014. STRUCTURE PLOT: A program for drawing elegant STRUCTURE bar plots in user friendly interface. Springerplus 3:431.

Rohlf, J.F. 1998. NTSYS: Numerical taxonomy and multivariate analysis system, version 2.02. Exeter Software, Setauket.

Temnykh, S., G. DeClerck, A. Lukashova, L. Lipovich, S. Cartinhour, and S. McCouch. 2001. Computational and experimental analysis of microsatellites in rice (Oryza sativa L.): Frequency, length variation, transposon associations, and genetic marker potential. Genome Res. 11:1441-1452.

Toth, G., Z. Gaspari, and J. Jurka. 2000. Microsatellites in different eukaryotic genomes: Survey and analysis. Genome Res. 10:967-981.

Tranbarger, T.J., W. Kluabmongkol, D. Sangsrakru, F. Morcillo, J.W. Tregear, S. Tragoonrung, and N. Billotte. 2012. SSR markers in transcripts of genes linked to posttranscriptional and transcriptional regulatory functions during vegetative and reproductive development of Elaeis guineensis. BMC Plant Biol. 12:1.
Van Geest, G., P.M. Bourke, R.E. Voorrips, A. Marasek-Ciolakowska, Y. Liao, A. Post, U. van Meeteren, R.G.F. Visser, C. Maliepaard, and P. Arens. 2017. An ultra-dense integrated linkage map for hexaploid chrysanthemum enables multi-allelic QTL analysis. Theor. Appl. Genet. 130:2527-2541.

Varshney, R.K., A. Graner, and M.E. Sorrells. 2005. Genic microsatellite markers in plants: Features and applications. Trends Biotechnol. 23:48-55.

Wang, Z., J. Li, Z. Luo, L. Huang, X. Chen, B. Fang, Y. Li, J. Chen, and X. Zhang. 2011. Characterization and development of ESTderived SSR markers in cultivated sweetpotato (Ipomoea batatas). BMC Plant Biol. 11:139.

Wang, H., J. Jiang, S. Chen, X. Qi, H. Peng, P. Li, A. Song, Z. Guan, W. Fang, Y. Liao, and F. Chen. 2013. Next-generation sequencing of the Chrysanthemum nankingense (Asteraceae) transcriptome permits large-scale unigene assembly and SSR marker discovery. PLoS One 8:E62293.

Wu, J., C. Cai, F. Cheng, H. Cui, and H. Zhou. 2014. Characterisation and development of EST-SSR markers in tree peony using transcriptome sequences. Mol. Breed. 34:1853-1866.

Xiang, X., Z. Zhang, Z. Wang, X. Zhang, and G. Wu. 2015. Transcriptome sequencing and development of EST-SSR markers in Pinus dabeshanensis, an endangered conifer endemic to China. Mol. Breed. 35:158.

Yang, W., B.J. Glover, G. Rao, and J. Yang. 2006. Molecular evidence for multiple polyploidization and lineage recombination in the Chrysanthemum indicum polyploid complex (Asteraceae). New Phytol. 171:875-886.
You, Y., D. Liu, H. Liu, X. Zheng, Y. Diao, X. Huang, and Z. Hu. 2015. Development and characterisation of EST-SSR markers by transcriptome sequencing in taro (Colocasia esculenta (L.) Schoot). Mol. Breed. 35:134.

Zalapa, J.E., H. Cuevas, H. Zhu, S. Steffan, D. Senalik, E. Zeldin, B. McCown, R. Harbut, and P. Simon. 2012. Using next-generation sequencing approaches to isolate simple sequence repeat (SSR) loci in the plant sciences. Amer. J. Bot. 99:193-208.

Zhang, F., S. Chen, F. Chen, W. Fang, and F. Li. 2010. A preliminary genetic linkage map of chrysanthemum (Chrysanthemum morifolium) cultivars using RAPD, ISSR and AFLP markers. Scientia Hort. 125:422-428.

Zhang, F., S. Chen, F. Chen, W. Fang, Y. Chen, and F. Li. 2011. SRAP-based mapping and QTL detection for inflorescence-related traits in chrysanthemum (Dendranthema morifolium). Mol. Breed. 27:11-23.

Zhang, Y., S. Dai, Y. Hong, and X. Song. 2014. Application of genomic SSR locus polymorphisms on the identification and classification of chrysanthemum cultivars in China. PLoS One 8:E104856.

Zhao, H.E., Z.H. Liu, X. Hu, J.L. Yin, W. Li, G.Y. Rao, X.H. Zhang, C.L. Huang, N. Anderson, Q.X. Zhang, and J.Y. Chen. 2009. Chrysanthemum genetic resources and related genera of Chrysanthemum collected in China. Genet. Resources Crop Evol. 56:937-946.

Zheng, J.S., C.Z. Sun, S.N. Zhang, X.L. Hou, and G. Bonnema. 2016. Cytogenetic diversity of simple sequences repeats in morphotypes of Brassica rapa ssp. chinensis. Front. Plant Sci. 7:1049. 


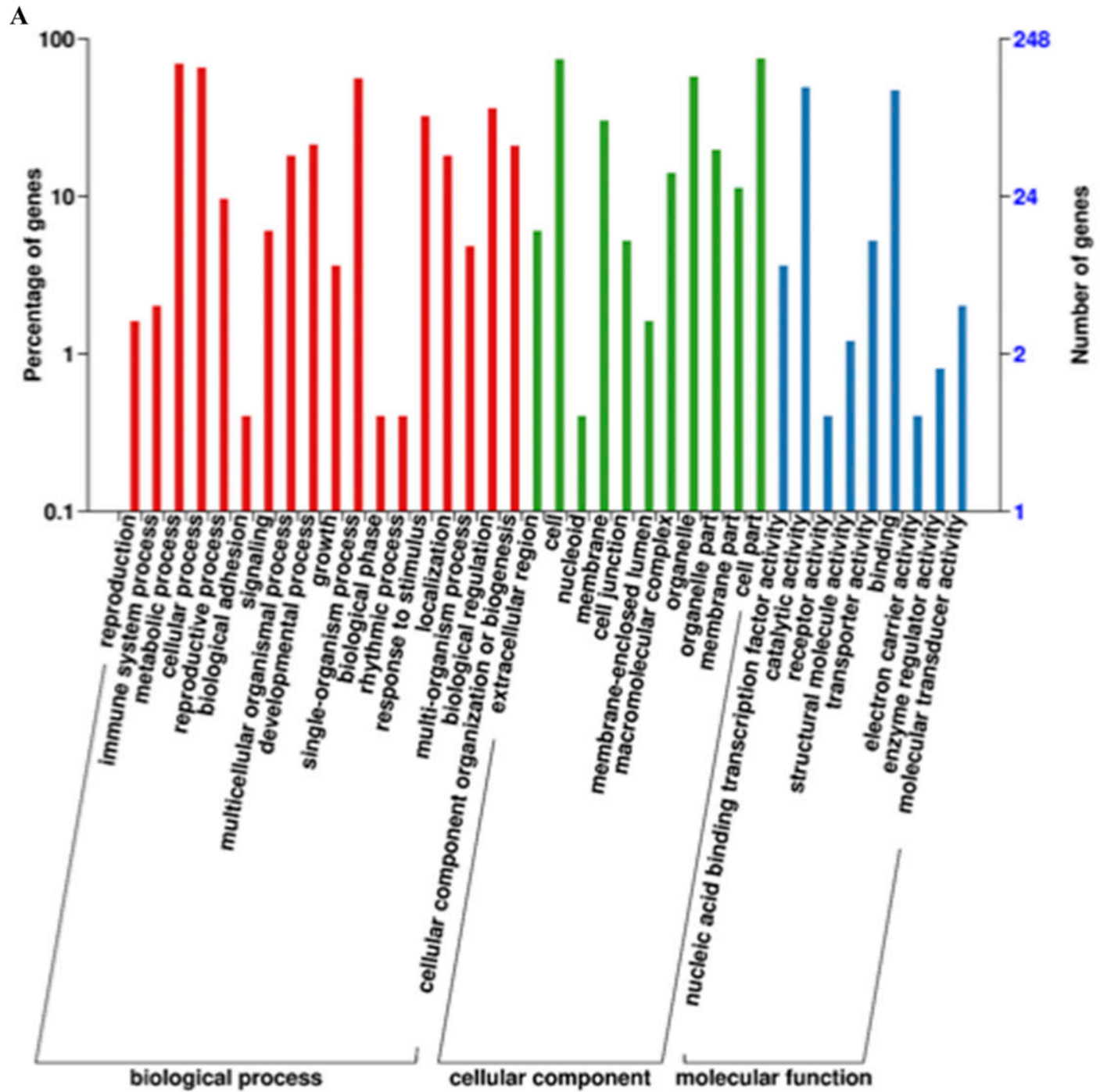

B

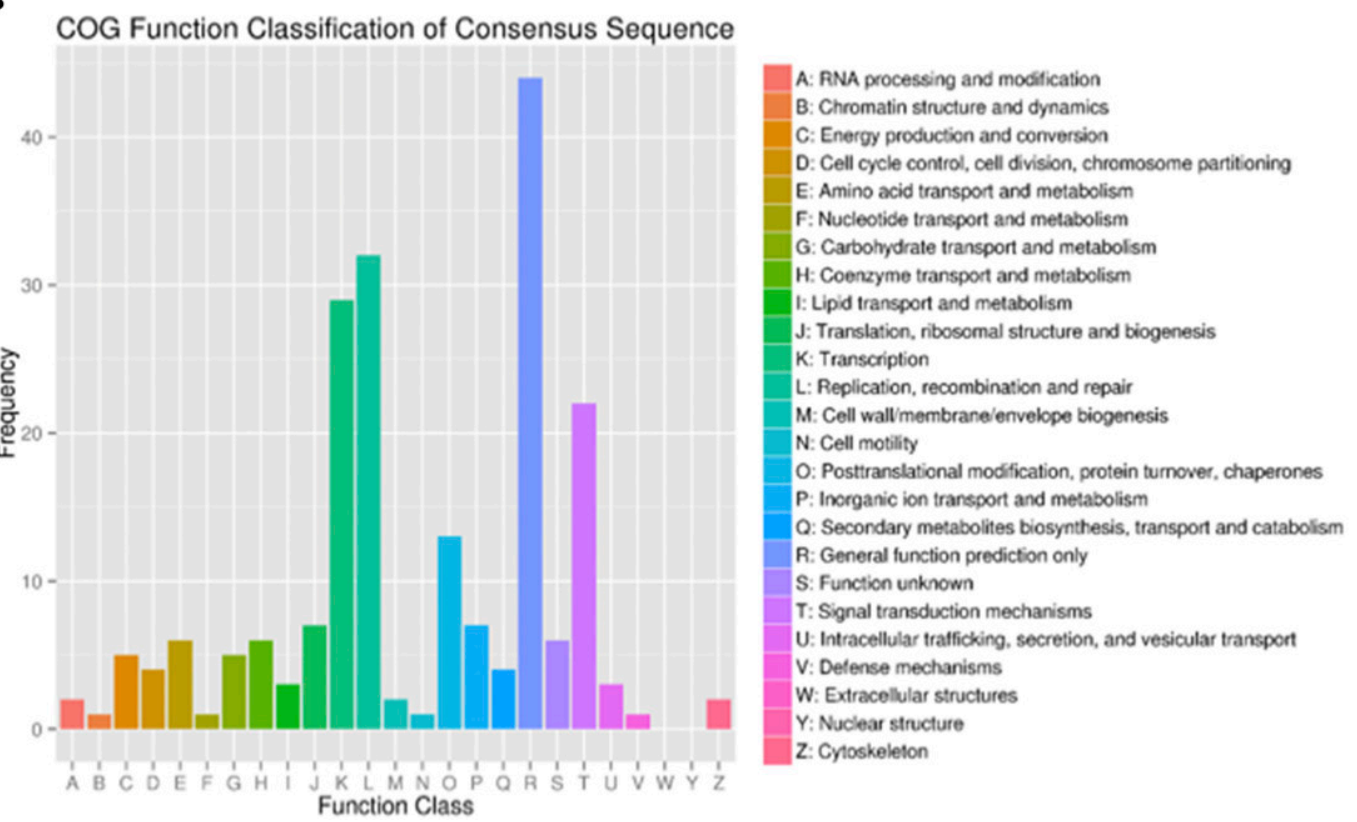

Supplemental Fig. 1. (A) The classification of 361 unigenes containing polymorphic microsatellite based on the Gene Ontology (GO) annotation. (B) The classification of 361 unigenes containing polymorphic microsatellite based on the clusters of orthologous groups (COG) annotation. (C) The top 10 enriched pathways involved the unigenes containing polymorphic microsatellite. mRNA = messenger RNA. 


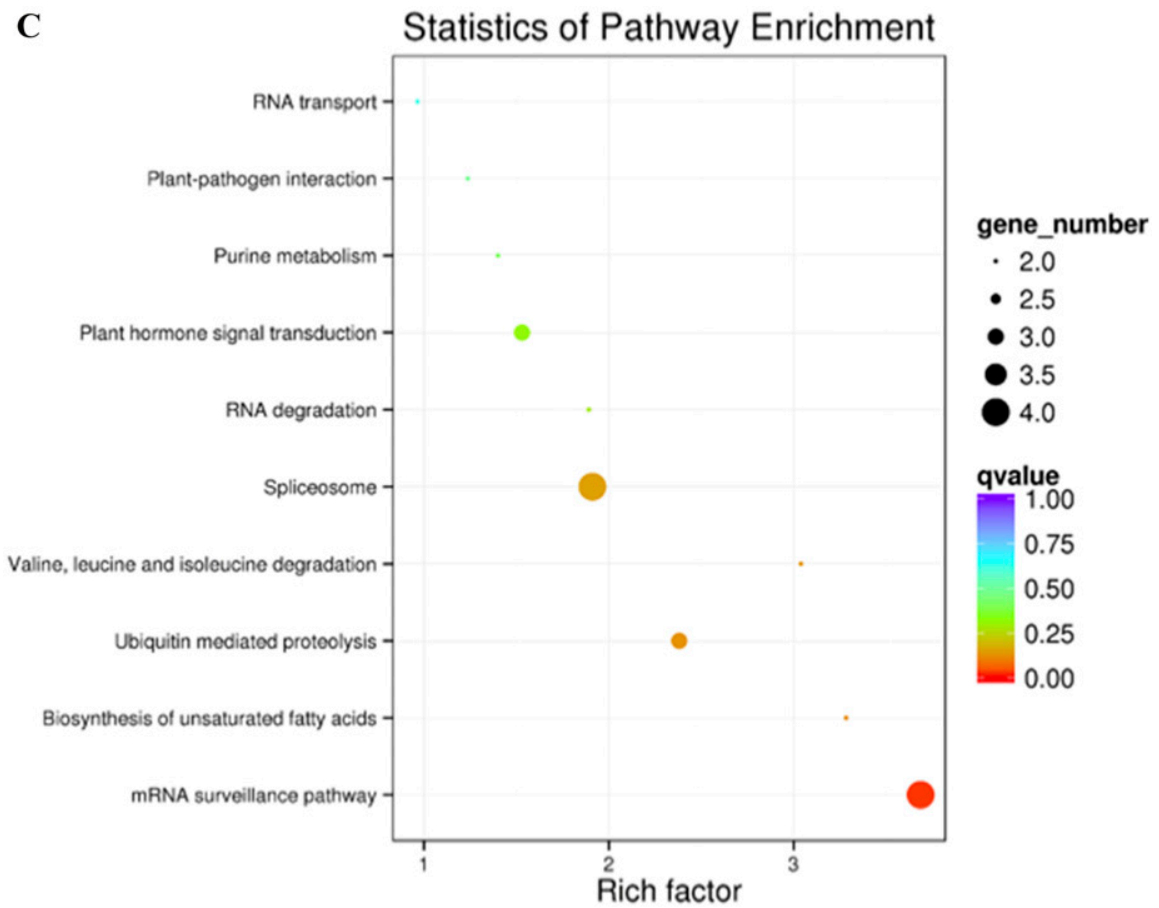

Supplemental Fig. 1. (Continued). 
Supplemental Table 1. Abbreviation and origin of 59 samples in this study.

\begin{tabular}{|c|c|c|c|c|c|}
\hline Code & Accession & Abbreviation & Ploidy & Species or cultivar types/origin & Notes \\
\hline 1 & Chrysanthemum indicum & ind1 & $2 \mathrm{x}$ & Species/Korea & \\
\hline 2 & Chrysanthemum chanetii & cha & $4 \mathrm{x}$ & Species/Beijing & \\
\hline 3 & Chrysanthemum nankingense & $\operatorname{man}$ & $2 \mathrm{x}$ & Species/Jiangsu Province & \\
\hline 4 & Chrysanthemum indicum & ind 2 & $4 \mathrm{x}$ & Species/Hubei Province & \\
\hline 5 & Chrysanthemum indicum var. aromaticum & ind 3 & $2 \mathrm{x}$ & Species/Hubei Province & \\
\hline 6 & Chrysanthemum zawadskii & zaw & $6 x$ & Species/Heilongjiang Province & \\
\hline 7 & Ajania fruticulosa & fru & & Species/Qinghai Province & \\
\hline 8 & Ajania potaninii & pot & & Species/Sichuan Province & \\
\hline 9 & Ajania achilloides & ach & & Species/Inner Mongolia & \\
\hline 10 & Ajania scharnhorstii & $\operatorname{sch}$ & & Species/Xinjiang Province & \\
\hline 11 & Ajania khartensisc & kha & & Species/Yunnan Province & \\
\hline 12 & Ajania purpurea & pur & & Species/Tibet & \\
\hline 13 & Ajania pacifica & pac 1 & $10 x$ & Species/Beijing & \\
\hline 14 & & pac 2 & & Species/Beijing & \\
\hline 15 & & pac 3 & & Species/Beijing & \\
\hline 16 & & pac 4 & & Species/Beijing & \\
\hline 17 & Chrysanthemum morifolium & chr1 & & Cultivars/Beihai Park in Beijing & Large chrysanthemum \\
\hline 18 & & $\operatorname{chr} 2$ & & Cultivars/Beihai Park in Beijing & Large chrysanthemum \\
\hline 19 & & $\operatorname{chr} 3$ & & Cultivars/Beihai Park in Beijing & Large chrysanthemum \\
\hline 20 & & $\operatorname{chr} 4$ & & Cultivars/Beihai Park in Beijing & Large chrysanthemum \\
\hline 21 & & $\operatorname{chr} 5$ & & Cultivars/Beihai Park in Beijing & Large chrysanthemum \\
\hline 22 & & chr6 & & Cultivars/Beihai Park in Beijing & Large chrysanthemum \\
\hline 23 & & $\operatorname{chr} 7$ & & Cultivars/Beihai Park in Beijing & Large chrysanthemum \\
\hline 24 & & $\operatorname{chr} 8$ & & Cultivars/Beihai Park in Beijing & Large chrysanthemum \\
\hline 25 & Ajania pacifica hybrids & aja1 & & Intergeneric hybrids/Beijing & \\
\hline 26 & & aja2 & & Intergeneric hybrids/Beijing & \\
\hline 27 & & aja3 & & Intergeneric hybrids/Beijing & \\
\hline 28 & & aja4 & & Intergeneric hybrids/Beijing & \\
\hline 29 & & aja5 & & Intergeneric hybrids/Beijing & \\
\hline 30 & & aja6 & & Intergeneric hybrids/Beijing & \\
\hline 31 & & aja7 & & Intergeneric hybrids/Beijing & \\
\hline 32 & & aja8 & & Intergeneric hybrids/Beijing & \\
\hline 33 & & aja9 & & Intergeneric hybrids/Beijing & \\
\hline 34 & & aja10 & & Intergeneric hybrids/Beijing & \\
\hline 35 & & aja11 & & Intergeneric hybrids/Beijing & \\
\hline 36 & & aja12 & & Intergeneric hybrids/Beijing & \\
\hline 37 & & aja13 & & Intergeneric hybrids/Beijing & \\
\hline 38 & Baiou & bo & & Cultivars/Beihai Park in Beijing & Large chrysanthemum \\
\hline 39 & Lvyishang & lys & & Cultivars/Beihai Park in Beijing & Large chrysanthemum \\
\hline 40 & Fanxing & fx & & Cultivars/Beihai Park in Beijing & Escarpment chrysanthemum \\
\hline 41 & Junziyu & jzy & & Cultivars/Beihai Park in Beijing & Escarpment chrysanthemum \\
\hline 42 & Shiyongju & syj & & Cultivars/Beijing Forestry University & Edible chrysanthemum \\
\hline 43 & Fenditan & fdt & & Cultivars/Beijing Forestry University & Groundcover chrysanthemum \\
\hline 44 & Pudifendai & pdfd & & Cultivars/Beijing Forestry University & Groundcover chrysanthemum \\
\hline 45 & Ziyunqinfang & zyqf & & Cultivars/Beijing Forestry University & Groundcover chrysanthemum \\
\hline 46 & Fanhuasijin & fhsj & & Cultivars/Beijing Forestry University & Groundcover chrysanthemum \\
\hline 47 & Zhaoyanghong & zyh & & Cultivars/Beijing Forestry University & Groundcover chrysanthemum \\
\hline 48 & Jinzhu & $\mathrm{jz}$ & & Cultivars/Beijing Forestry University & Groundcover chrysanthemum \\
\hline 49 & Nongfenzhaoxia & nfzx & & Cultivars/Beijing Forestry University & Groundcover chrysanthemum \\
\hline 50 & Baisha & bs & & Cultivars/Beijing Forestry University & Groundcover chrysanthemum \\
\hline 51 & Danhanfen & dhf & & Cultivars/Beijing Forestry University & Groundcover chrysanthemum \\
\hline 52 & Jinjihongling & jjhl & & Cultivars/Beijing Forestry University & Groundcover chrysanthemum \\
\hline 53 & Maoxiangyu & mxy & & Cultivars/Beijing Forestry University & Groundcover chrysanthemum \\
\hline 54 & Xiangfei & $\mathrm{xf}$ & & Cultivars/Beijing Forestry University & Groundcover chrysanthemum \\
\hline 55 & Mibaizao & $\mathrm{mbz}$ & & Cultivars/Beijing Forestry University & Groundcover chrysanthemum \\
\hline 56 & Pudidanfen & pddf & & Cultivars/Beijing Forestry University & Groundcover chrysanthemum \\
\hline 57 & Yulong & yl & & Cultivars/Beijing Forestry University & Groundcover chrysanthemum \\
\hline 58 & HJJ & hjj & & Cultivars/Beijing Forestry University & \\
\hline 59 & $\mathrm{SN} * \mathrm{MXY}$ & sm & & Cultivars/Beijing Forestry University & \\
\hline
\end{tabular}




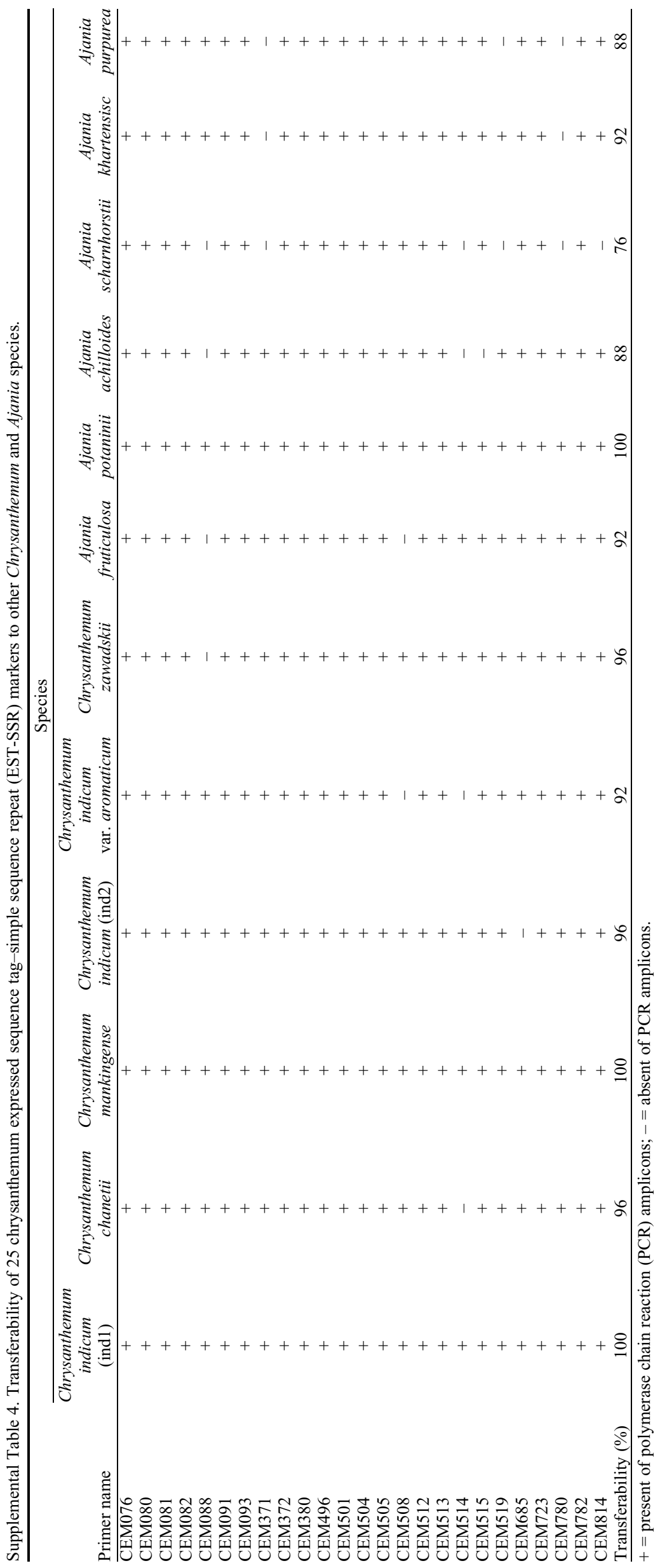

